\title{
Avaliação das condições de armazenamento de medicamentos em domicílios do município de Uruguaiana - RS
}

\author{
Rodrigo de Souza Balk', Odete Messa Torres², Taciane Maia Barbosa ${ }^{3}$, \\ Gabriel de Paula Gollino ${ }^{4}$, Luciana Fatima Santos Chies $^{5}$
}

\section{RESUMO}

Introdução: 0 armazenamento adequado e a preservação de medicamentos são fatores fundamentais para sua eficácia. A vasta oferta de medicamentos, a falta da atenção farmacêutica referente ao modo de armazenamento dos mesmos e a má conservação podem afetar as características do fármaco e o acúmulo de medicamentos em domicilios pode trazer riscos à saúde. Objetivo: Avaliar através de visitas domiciliares as condições dos medicamentos armazenados nos domicílios de usuários atendidos pelo Programa Práticas Integradas em Saúde Coletiva. Metodologia: Estudo qualitativo e quantitativo realizado em 20 domicílios atendidos pelo Programa Práticas Integradas em Saúde Coletiva (PISC) no município de Uruguaiana- RS os quais foram aplicados um questionário estruturado. As variáveis dos questionários avaliados foram: distribuição sóciodemográfica, locais e condições de armazenamento dos medicamentos. Resultados: Sobre a distribuição sóciodemográfica observou-se que a população compreendeu $85 \%$ sexo feminino e $15 \%$ sexo masculino com idade média dos entrevistados de 56,7. Em todos os domicílios visitados foram encontrados estoques de medicamentos e todos os usuários estavam em tratamento farmacológico. Observou-se a ausência da embalagem primária em maior parte dos medicamentos estocados e nos 20 domicílios visitados a média de medicamentos estocados foi de 7,3 sendo a maioria do gênero anti-hipertensivo, hipoglicemiante oral e diurético. Conclusão: Torna-se indispensável a orientação adequada para cumprimento da dosagem, a influência dos alimentos, a interação com outros medicamentos, o reconhecimento de reações adversas potenciais e as condições de conservação do medicamento reduzindo assim o alto índice de medicamentos armazenados nos domićilios de maneira inadequada propiciando risco a qualidade de vida

Descritores: Qualidade de Vida; Domicílio; Medicamentos; Armazenamento

\section{Evaluation of drug storage conditions in houses in the city of Uruguaiana - RS}

\begin{abstract}
Introduction: Proper storage and preservation of drugs are critical factors for its effectiveness. A wide range of medicines, lack of pharmaceutical regarding storage so the same attention and poor maintenance can affect the drug's characteristics and the accumulation of drugs can bring health risks. Objective: To evaluate through home visits the conditions of the medications stored in the homes of users served by the Integrated Programme in Public Health Practice. Methodology: Qualitative and quantitative study conducted in 20 households served by Integrated Program in Public Health Pratice (PISC) in the municipality of Uruguaiana- RS which were administered a structured questionnaire. The variables of the evaluated questionnaires were: socio-demographic distribution, local and medication storage conditions. Results: On the socio-demographic distribution was observed that the population comprised $85 \%$ female and $15 \%$ male with an average age of respondents was 56.7. In all households visited were found drugs stocks and all members were on pharmacological treatment. There was a lack of primary packaging in most stocked drugs and 20 homes visited an average of stocked medicines was 7.3 and most of the anthypertensive gender, oral hypoglycemic and diuretic. Conclusion: It is essential to the proper guidance to comply with the dosage, the influence of food, interaction with other medications, recognition of potential side effects and drug storage conditions thereby reducing the high rate of drugs stored in homes so inadequate that undermines the effectiveness of the drug or even risk bringing the quality of life.
\end{abstract}

Descriptors: Quality of Life; Home; Medications; Storage.

${ }^{1}$ Doutor em Ciências Biológicas pela Universidade Federal de Santa Maria (UFSM), Santa Maria, RS, Brasil.

${ }^{2}$ Doutora em Enfermagem pela Universidade Federal de São Paulo (UNIFESP), São Paulo, SP, Brasil.

${ }^{3}$ Graduada em Farmacia pela Universidade Federal do Pampa (UNIPAMPA), Uruguaiana, RS, Brasil.

${ }^{4}$ Graduado em Farmacia pela Universidade Federal do Pampa (UNIPAMPA), Uruguaiana, RS, Brasil.

${ }^{5}$ Acadêmica de Enfermagem na Universidade Federal do Pampa (UNIPAMPA), Uruguaiana, RS, Brasil. 


\section{Introdução}

Medicamentos são produtos especiais elaborados com a finalidade de diagnosticar, prevenir, curar doenças ou aliviar seus sintomas, sendo produzidos com rigoroso controle técnico para atender às especificações determinadas pela Agência Nacional de Vigilância Sanitária - ANVISA1. Possuem um papel cada vez mais relevante e estão presentes na maioria dos procedimentos terapêuticos, sendo usados como instrumento de prevenção e tratamento às doenças. Ao mesmo tempo, a frequência de uso, a facilidade de aquisição e a publicidade sem restrições banalizaram a utilização de medicamentos, desconsiderando os potenciais riscos de uso sem orientações ${ }^{2}$. Os medicamentos devem ser dispensados de acordo com a necessidade do solicitante, garantindo adequadas condições de transporte, rastreabilidade do produto e a preservação da identificação até o consumidor final ${ }^{3}$. A denominada cultura da pílula, que intensifica o fenômeno da medicalização, é entendida como a crescente e elevada dependência dos indivíduos e da sociedade em relação a oferta de serviços e bens de ordem médico-assistencial e seu consumo cada vez mais intensivo, em frequência muitas vezes desnecessária, colocando o medicamento como sinônimo de cura e/ou de saúde ${ }^{4,5}$.

A segurança de um medicamento inicia com o balanço de seu inerente potencial de risco, sendo atribuída corretas prescrições (doses, intervalos, horários, duração), dispensação, aquisição (qualidade, boas práticas de fabricação), administração (diluições, aplicações, assepsia nas injeções, horários, alimentos concomitantes), armazenamento (umidade, temperatura, tempo de validade) e termina com a adesão do paciente ao tratamento. A falta de adesão pode ser apontada como um motivo de acúmulo de medicamentos nos domicílios ${ }^{6}$.

Armazenar medicamentos nos domicílios tornou-se uma prática comum, podendo representar um potencial risco para o surgimento de agravos a saúde ${ }^{2}$. Os locais ${ }^{7}$ mais comuns de armazenamento de medicamentos são gavetas, dispensas, pias, dentro de caixas ou de armários e ignoram o tempo de armazenamento depois de aberto, assim como a sua exposição a altas temperaturas, luz solar ou artificial e umidade.

A Organização Mundial da Saúde (OMS) e, no Brasil, a Política Nacional de Medicamentos, preconizam que 0 uso racional de medicamentos ocorre quando os pacientes recebem a medicação adequada às suas necessidades clínicas, nas doses correspondentes aos seus requisitos individuais, durante um período de tempo adequado e ao menor custo possível para si e para a comunidade ${ }^{8,9}$. Para que ocorra o uso racional de medicamentos é necessária atenção farmacêutica adequada aos pacientes. A atenção farmacêutica é uma prática profissional que visa orientar 0 paciente sobre o uso racional de medicamentos proporcionando-lhe qualidade de vida por meio de ações do profissional farmacêutico, bem como de suas condutas, compromisso, responsabilidade, valores éticos e conhecimento ${ }^{10}$.

A visita domiciliar é uma "forma de atenção em Saúde Coletiva voltada para o atendimento ao indivíduo, à família ou a coletividade que é prestada aos domicílios ou junto aos diversos recursos sociais locais, visando à equidade da assistência em saúde ${ }^{11}$ e pode ser entendida como atendimento realizado por profissional ou equipe de saúde no domicilio do usuário, com objetivo de avaliar as necessidades deste, de seus familiares e do ambiente onde vive, para estabelecer um plano assistencial voltado à recuperação ou reabilitação e as orientações são feitas para as pessoas responsáveis pela continuidade do cuidado no domicílio ${ }^{12}$. É importante considerar que neste ambiente, a família participa ativamente das definições de padrões de cuidado, além da participação na definição da saúde ${ }^{13}$.

Mediante a vasta oferta de medicamentos aliada às inúmeras propagandas no meio farmacêutico, o descaso com os medicamentos, a falta de informação referente ao modo de armazenamento dos mesmos e sabendo-se que a má conservação podem afetar as características do fármaco e que o acúmulo de medicamentos em domicílio pode trazer sérios riscos a saúde humana, este estudo propõe realizar uma avaliação a fim de conhecer as condições e características dos medicamentos armazenados nos domicílios de pacientes atendidos pelo Programa Práticas Integradas em Saúde Coletiva - PISC no município de Uruguaiana-RS.

\section{Metodologia}

Este trabalho foi submetido para apreciação junto ao Comitê de Ética em Pesquisa (CEP) da Universidade Federal do Pampa e somente após aprovação do CEP com parecer número 756.020 iniciaram as entrevistas. Cada usuário recebeu o termo de consentimento livre e esclarecido (TCLE), para a participação na pesquisa. 
Com a inserção da Universidade Federal do Pampa (UNIPAMPA) na cidade de Uruguaiana, busca-se o compromisso social que a mesma desenvolve. Desta forma e por meio da Universidade, no ano de 2009 foi criado o Projeto de Extensão Universitária Práticas Integradas em Saúde Coletiva (PISC), de iniciativa discente e na sua composição contando com cursos de enfermagem, farmácia e fisioterapia. No ano de 2010 houve a ampliação da proposta transformando-o em um amplo programa multi e interdisciplinar apresentando como foco a atenção básica em saúde. Dentre as diversas atividades realizadas estão às visitas domiciliares, no qual, os acadêmicos, o professor supervisor e a equipe de atenção básica adstrita orientam os cuidados domiciliares aos usuários de diferentes UBS.

A escolha dos domicílios foi realizada após discussão com a equipe de saúde sobre usuários necessitados de atenção domiciliar integrada de enfermagem, farmácia e fisioterapia e a escolha dos usuários através da identificação, pelas Agentes Comunitárias de Saúde, em cada micro área do território adscrito das UBS atendidas. As visitas domiciliares foram realizadas pela equipe de acadêmicos acompanhados dos agentes comunitários de saúde e supervisão docente.

Esta pesquisa foi realizada entre os meses de junho e agosto de 2014 e tem delineamento prospectivo de caráter quantitativo e qualitativo. Participaram do estudo acompanhantes dos usuários e/ou usuários atendidos pelo PISC em visitas domiciliares nas residências localizadas próximas das Unidades Básicas de Saúde (UBS) 07, 14, 19 no município de Uruguaiana-RS atingindo 20 domicílios repassados pelos enfermeiros responsáveis de cada UBS, sendo o critério de escolha a partir de usuários hipertensos e diabéticos com dificuldades em fazer o tratamento terapêutico adequado. $O$ fator de inclusão do estudo era que o usuário possuísse doenças crônicas como HAS e DM. Nestes usuários/acompanhantes foi aplicado um questionário composto de perguntas fechadas envolvendo distribuição sociodemográfica, locais e condições de armazenamento dos medicamentos. Este questionário é baseado nas necessidades para o cuidado em saúde, e orientações de armazenamento e administração de medicamentos ${ }^{2}$. Após a seleção dos domicílios, os acompanhantes ou usuários passaram por uma entrevista com perguntas abertas que teve como finalidade o esclarecimento dos objetivos e procedimentos do protocolo.

\section{Procedimentos Experimentais (Instrumento de Avaliação)}

O questionário foi dividido em três etapas:

Primeiro momento: identificação e avaliação das características sóciodemográficas tais como, responsável pelos medicamentos no domicílio, idade, sexo, grau de escolaridade do responsável pelos medicamentos, número de pessoas que residem na casa, número de cômodos existentes no domicílio, número de crianças residindo no local e a renda mensal da família.

Segundo momento: identificação do local de armazenamento dos medicamentos, se os entrevistados possuíam medicamentos estocados em casa, em quais cômodos os medicamentos são armazenados, se existe limpeza periódica do local de armazenamento e/ou dos medicamentos, se o entrevistado já identificou algum tipo de inseto ou roedor entre os medicamentos, controle periódico da data de validade dos medicamentos, onde o entrevistado costuma adquirir os medicamentos, se a família já recebeu orientação sobre como armazenar seus medicamentos em casa.

Terceiro momento: observação, pelo entrevistador, das condições de armazenamento, exposição dos medicamentos à luz, à umidade, ao calor, limpeza, armazenamento em recipiente com tampa, armazenamento em local fora do alcance de crianças, número de medicamentos estocados, existência ou não de medicamentos vencidos, quantidade e se estes se encontravam armazenados em suas embalagens originais.

Os resultados da entrevista foram analisados por estatística descritiva utilizando o programa Excel para Windows.

\section{Resultados}

Foram visitados 20 domicílios de usuários diabéticos e hipertensos, 85\% dos usuários eram do sexo feminino e 15\% sexo masculino de um total de 56 usuários entrevistados, cadastrados nas UBSs parceiras do PISC. À idade média dos entrevistados foi de 56,7 com mínima de 50 anos e máxima de 80 anos. Maior parte dos entrevistados possuía ensino fundamental incompleto (Tabela 1). 
Tabela 1 - Características sociodemográficas dos usuários cadastrados nas UBSs no município de Uruguaiana-RS, parceiras do Programa Práticas Integradas em Saúde Coletiva.

\begin{tabular}{c|c|c}
\hline Características & Número de citações & $\%$ \\
\hline Grau de escolaridade & & 20 \\
\hline Nenhuma & 4 & 50 \\
\hline Fundamental Incompleto & 10 & 10 \\
\hline Fundamental Completo & 2 & 15 \\
\hline Ensino Médio & 3 & 0 \\
\hline Ensino Superior Incompleto & 1 & \\
\hline Ensino Superior & 0 & \\
\hline Média de pessoas residentes no domicílio & 2.8 & 20 \\
\hline Média de cômodos & 4,6 & 65 \\
\hline Média de Crianças no domicílio & 0,55 & 0 \\
\hline Renda familiar & & 15 \\
\hline$<1$ salário mínimo & 4 & \\
\hline Entre 1 e 2 salários & 13 & \\
\hline Entre 2 e 3 salários & 0 & \\
\hline Maior que 3 salários & 3 & \\
\hline
\end{tabular}

Em todos os domicílios visitados foram encontrados estoques de medicamentos e todos os usuários estavam em tratamento farmacológico. $\mathrm{O}$ armazenamento de medicamentos na maioria das residências é inadequado e em mais de um cômodo na maioria dos domicílios visitados (Tabela 2).

Tabela 2 - Local de armazenamento dos medicamentos

\begin{tabular}{c|c|c}
\hline \multirow{2}{*}{ Cômodo } & Local & Número de citações \\
\hline \multirow{2}{*}{ Cozinha } & Prateleira & 7 \\
\cline { 2 - 3 } & Armário & 4 \\
\hline \multirow{2}{*}{ Quarto } & Comoda & 5 \\
\cline { 2 - 4 } & Roupeiro & 3 \\
\hline Sala & Estante & 0 \\
\hline Banheiro & Balcão & 0 \\
\hline Outros & & \\
\hline
\end{tabular}

Observou-se a ausência da embalagem primária em maior parte dos medicamentos estocados, visto que os medicamentos encontrados nessas condições são adquiridos nas Unidades Básicas que realizam a distribuição dos mesmos sem a embalagem primária. Porém os medicamentos encontravam-se em suas embalagens secundárias, ou seja, em cartelas quando formas sólidas, ou frascos quando em formas líquidas. Foi observado a falta de orientação para os usuários no momento da prescrição ou entrega dos medicamentos, o que ocasiona exposições, controle e administração dos medicamentos de forma inadequada (Tabela 3). 
Tabela 3 - Condições de armazenamento que os medicamentos se encontravam.

\begin{tabular}{c|c|c}
\hline Características & Sim (\%) & Não (\%) \\
\hline Exposto a luz & 35 & 65 \\
\hline Exposto a unidade & 40 & 45 \\
\hline Exposto ao calor & 55 & 35 \\
\hline Limpos e sem poeira & 65 & 80 \\
\hline Em recipiente com tampa & 20 & 0 \\
\hline Medicamentos estocados & 100 & 85 \\
\hline Medicamentos vencidos & 15 & 80 \\
\hline Embalagem primaria & 20 & 20 \\
\hline Embalagem secundaria & 80 & 60 \\
\hline Recebe orientações sobre armazenamentos & 40 & 5 \\
\hline Controle da validade & 95 & \\
\hline
\end{tabular}

Nos 20 domicílios visitados a média de medicamentos estocados foi de 7,3 com mínimo de 1 e máximo de 27, sendo a maioria dos medicamentos dos gêneros anti-hipertensivo, hipoglicemiante oral e diurético. Segundo observações feitas pelos pesquisadores, alguns usuários possuíam pressão arterial e glicemia elevada, pois não costumavam fazer uso dos medicamentos nos horários corretos, devido à falta de orientação.

\section{Discussão}

Nas regiões de quatro UBSs do município de Uruguaiana/RS, os 20 usuários entrevistados (100\%) possuíam estoques de medicamentos, em média 7,3 por domicílio. Em um estudo realizado na cidade de ljuí/RS, com amostra de 321 usuários entrevistados, verificou-se em média 4,2 medicamentos por domicílio. Por outro lado, quando comparados a outro estudo realizado no município de Porto Alegre/RS observou-se o acúmulo de 20 medicamentos por domicílio em uma amostra de 101 entrevistados ${ }^{15}$. As divergências entre as médias encontradas pelos estudos citados podem estar relacionadas com a diferença no perfil socioeconômico dos entrevistados, pois em concordância com esse estudo, o primeiro avaliou uma população com baixo poder aquisitivo, onde os medicamentos eram adquiridos junto ao Sistema Único de Saúde (SUS), enquanto que o bairro no qual foram realizadas as entrevistas do segundo estudo, em Porto Alegre, possui uma população de maior poder aquisitivo, o que possibilita a compra deliberada de medicamentos.

Dos 20 domicílios analisados, observou-se que a maioria dos medicamentos estava armazenada de forma inadequada. Identificou-se que os locais de preferência para armazenamento foram quarto e cozinha, o que possivelmente se deve à acessibilidade do local ${ }^{15}$. Dos nove locais de armazenamento investigados, em $35 \%$ os medicamentos estavam expostos à luz, $40 \%$ à umidade e $55 \%$ ao calor. Tais fatores ocasionam a aceleração de diversos mecanismos de degradação das moléculas dos fármacos, o que é de extrema preocupação, pois compromete a estabilidade e ocasiona ineficiência na farmacoterapia do usuário ${ }^{16}$. Estes problemas podem ser reduzidos, ou até solucionados se houver orientação farmacêutica quanto aos locais de armazenamento, ou a leitura das especificações contidas nas bulas ${ }^{17}$, porém, a maioria dos medicamentos encontrados nos domicílios é dispensado pelo SUS e o sistema disponibiliza a medicação com ausência da sua embalagem primária e bula ${ }^{18}$.

Outro fator analisado foi o controle do prazo de validade dos medicamentos por parte dos entrevistados, nos quais $95 \%$ destes, realiza analise periódica da validade e 5\% não realiza. Apesar de ser uma pequena parcela, este resultado demonstra uma prática inadequada das unidades de dispensação e dispensadores em não orientar os usuários a analisarem os prazos de validade. Medicações com o prazo de validade vencido não podem ser utilizadas, pois podem provocar efeitos indesejáveis, diferentes das suas indicações terapêuticas originais ${ }^{19}$.

A maioria dos medicamentos encontrados nos domicílios foi dos gêneros anti-hipertensivo, hipoglicemiante oral e diurético, pois o fator de inclusão do estudo é que o usuário seja portador de alguma doença crônica, como HAS e DM. Entretanto, um outro estudo realizado nos municípios de Limeira e Piracicaba no estado de São Paulo com amostra aleatória simples, apontou a presença mais frequente de analgésicos/antipiréticos e antibióticos para uso sistêmico nos domicílios ${ }^{20}$. 
Diabetes Mellitus e Hipertensão Arterial Sistêmica fazem parte de uma realidade preocupante, com um percentual de 22,3\% a 43,9\% da população adulta brasileira apresentando a ocorrência de HAS ${ }^{21}$ e segundo estimativas da Organização Mundial de Saúde (OMS), o número de portadores de DM no ano de 2000 no mundo era de 177 milhões, no ano de 2014 cerca de 350 milhões de pessoas sofrem $\mathrm{DM}^{22,23}$.

De acordo com este estudo, constatou-se a importância de um farmacêutico vinculado as UBSs, para a prática de dispensação de medicamentos. No município deUruguaiana/RS esta prática érealizada porqualquer integrante daequipe, seja enfermeiros, assistentes sociais e ate mesmo atendentes, não sendo funções de tais categorias. A dispensação inadequada ocorre pois não há a presença farmacêuticos nas UBS's. Segundo a Lei do Exercício Profissional de Enfermagem ${ }^{24}$ e seu Decreto Regulamentador ${ }^{25}$, direcionam as atividades da profissão quanto ao cuidado e assistência de enfermagem, 0 que se refere à manipulação de medicamentos, cabe ao farmacêutico o preparo e administração das drogas. Dessa forma, tornase indispensável à presença de um farmacêutico para a orientação adequada do cumprimento da dosagem, a influência dos alimentos, a interação com outros medicamentos, o reconhecimento de reações adversas potenciais e as condições de conservação do medicamento26. Isso pode reduzir o alto índice de medicamentos armazenados nos domicílios de maneira inadequada, o que prejudica a efetividade do fármaco ou pode trazer riscos à qualidade de vida.

\section{Referências Bibliográficas}

1. Brasil. Agência Nacional de Vigilância Sanitária - Cartilha "O Que Devemos Saber Sobre Medicamentos". Agência Nacional de Vigilância Sanitária; 2010.

2. Valério WL. Avaliação do estoque domiciliar de medicamentos em um bairro do município de Forquilhinha-SC. 2009. [54] f. TCC (Graduação em Farmácia) - Universidade do Extremo Sul Catarinense, Criciúma. 2009.

3. Marin N, Luiza VL, Osorio De Castro CGS, Santos SM. (Org.). Assistência farmacêutica para gerentes municipais. Rio de Janeiro: OPAS, 2003, p. 373.

4. Barros JAC. Políticas farmacêuticas: a serviço dos interesses da saúde? Brasília: Unesco; 2004.

5. Lima GB, Nunes CCL, Barros CAJ. Uso de medicamentos armazenados em domicílio em uma população atendida pelo Programa Saúde da Família. Ciênc. Saúde coletiva 2010, vol.15, suppl.3, pp. 3517-3522.

6. Wannmacher L. Erros: evitar o evitável; Uso Racional de Medicamentos: Temas Selecionados 2005. 2 (7): 1-6.

7. Serafim EOP, Vecchio A, Gomes J, Miranda A, Moreno AH, Loffredo LMC et al. Qualidade dos medicamentos contendo dipirona encontrados nas residências de Araraquara e sua relação com a atenção farmacêutica. Rev. Bras. Cienc. Farm. 2007; Brasil, vol.43, n.1, pp. 127-135.

8. BRASIL, Ministério da Saúde, Secretaria de Políticas de Saúde. Política nacional de medicamentos. Rev Saude Publica. 2000;34(2):206-9.

9. Organización Mundial de la Salud. Perspectivas políticas sobre medicamentos de la OMS. Promoción del uso racional de medicamentos: componentes centrales.

10. OPAS - Organização Panamerica da Saúde. El Papel del Farmacéutico en el Sistema de Atención de Salud. Tokio, OPS/HSS/HSE/95.1, 1995.

11. Ceccim RB, Machado, NM. Contato Domiciliar em Saúde Coletiva. 1995. Porto Alegre: Universidade Federal do Rio Grande do Sul, s/d. 7p.

12. Fabricio SCC, Wehbe G, Nassur FB, Andrade Jl. Assistência domiciliar: a experiência de um hospital privado do interior paulista. Rev Lat Amer Enf. 2004;12(5):721-26.

13. Klock AD, Heck RM, Casarim ST. Cuidado Domiciliar: A Experiência da Residência Multiprofissional em Saúde da Família/UFPEL-MS/BID. Texto Contexto Enferm 2005; 14(2):237-45.

14. Bueno CS, Weber D, Oliveira KR. Farmácia caseira e descarte de medicamentos no bairro Luiz Fogliatto do município de ljuí - RS. Rev Ciênc Farm Básica Apl. 2009; 30(2):203-210.

15. Schenkel EP, Fernándes LC, Mengue SS. Como são armazenados os medicamentos nos domicílios? Acta Farm. Bonaerense 2005; 24 (2): 266-70.

16. Prista LVN, Alves AC, Morgado R. Tecnologia farmacêutica. Lisboa, Portugal: Fundação Calouste Gulbenkian, 2008. 3 v. 5 ed 
17. Leite EG. Estabilidade: importante parâmetro para avaliar a qualidade, segurança e eficácia de fármacos e medicamentos. 2006. 178p. Dissertação (Mestrado em Ciências Farmacêuticas). Programa de Pós-graduação em Ciências Farmacêuticas, Mestrado Profissionalizante em desenvolvimento Tecnológico e Controle de Qualidade de Produtos Farmacêuticos, UFRGS. Porto Alegre.

18. Lima GB, Araujo EJF, Sousa KMH, Benvido RF, Silva WCS, Correa RAC et al. Avaliação da utilização demedicamentos armazenados em domicílios por uma população atendida pelo PSF. Rev. Bras. Farm. 2008; 89 (2): 146 - 149.

19. Agência Nacional de Vigilância Sanitária. Medicamentos - Venda de medicamentos sem prescrição médica. Brasília: Agência Nacional de Vigilância Sanitária 2008. [online]. 2008 [acesso 2014 Ago 21]. Disponível em: http://www.anvisa. gov/profissional/medicamentos/farmacia_caseira.htm\#

20. Tourinho FSV, Bucaretchi F, Stephan C, Cordeiro R. Farmácias domiciliares e sua relação com a automedicação em crianças e adolescentes. J. Pediatr. (Rio J.) 2008; Porto Alegre, v. 84; n. 5; pp. 416-422.

21. Ministério da Saúde. Secretaria de Atenção à Saúde. Departamento de Atenção Básica. Hipertensão arterial sistêmica para o Sistema Único de Saúde. Brasília: Ministério da Saúde; 2006. 58 p.

22. Ministério da Saúde. Secretaria de Atenção à Saúde. Departamento de Atenção Básica. Diabetes Mellitus para 0 Sistema Único de Saúde. Brasília: Ministério da Saúde; 2006; p. 9.

23. Organização Mundial de Saúde. Diabetes Afeta Cerca de 350 Milhões de Pessoas no Mundo. OMS, 2014.

24. Lei $n^{0} 7.498$, de 25 de junho de 1986. Dispõe sobre a regulamentação do exercício da Enfermagem e dá outras providências.

25. Decreto $n^{\circ} 94.406$, de 08 de junho de 1987. Regulamentada a Lei $n^{\circ} 7.498$, de 25 de junho de 1986. Dispõe sobre o exercício da Enfermagem, e dá providências. Disponível em: http://www.planalto.gov.br/ccivil_03/decreto/1980-1989/ D94406.htm. Acesso em 06 de Agosto de 2014.

26. Ministério da Saúde. Secretaria de Políticas de Saúde. Departamento de Formulação de Políticas de Saúde. Política nacional de medicamentos 2001/Ministério da Saúde, Secretaria de Políticas de Saúde, Departamento de Atenção Básica. -Brasília: Ministério da Saúde, 2001; pp. 34.

\section{Rodrigo de Souza Balk}

Endereço para correspondência - Universidade Federal do Pampa, BR 472, KM 592, Caixa Postal 118, CEP: 97508-000, Uruguaiana, RS, Brasil.

E-mail: rodrigo.balk@gmail.com

Lattes: http://lattes.cnpq.br/8871615237315962

Odete Messa Torres - odetetorres@gmail.com

Taciane Maia Barbosa - bm.taciane@gmail.com

Gabriel de Paula Gollino - ggollino@gmail.com

Luciana Fátima Santos Chies - luchies_21@hotmail.com

\section{Enviado em 24 de agosto de 2015. Aceito em 22 de outubro de 2015.}


